\title{
Microanalysis surface studies and photoemission properties \\ of CsI photocathodes
}

\author{
J. Almeida1), A. Braem2), A. Breskin3), A. Buzulutskov3), R. Chechik3)* \\ S. Cohen ${ }^{3)}$, C. Coluzza1), E. Conforto'), G. Margaritondo'), E. Nappi4), G. Paic ${ }^{5)}$, \\ F. Piuz'), T. dell'Orto1), T. Scognetti4) S. Sgobba'2), and B.P. Tonner6) \\ 1) EPFL, Lausanne, Switzerland \\ 2) CERN, Geneva, Switzerland \\ 3) The Weizmann Institute, Rehovot, Israel \\ 4) INFN, Bari, Italy \\ 5) SUBATECH, Nantes, France \\ 6) SR Center, Univ. of Wisconsin-Madison, Stougton, USA.
}

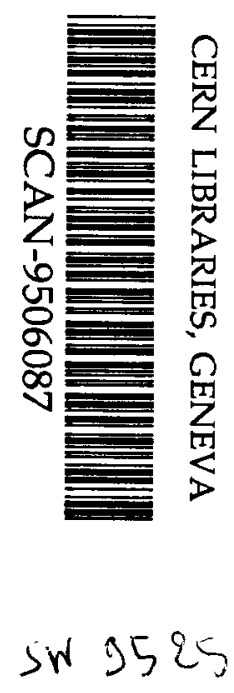

\begin{abstract}
We present recent results of the study of surface properties and quantum efficiency (QE) of CsI photocathodes prepared on various substrates. Microanalysis methods provide laterally resolved surface morphology and chemical composition of the photoemissive film. Integral measurements of the QE of CsI were done with a monochromator system and a RICH device. It was shown that CsI films deposited on large area $\mathrm{Ni}$ - or $\mathrm{Ni} / \mathrm{Au}$-coated printed circuit electrodes have a uniform crystalline structure and an average $\mathrm{QE}$ close to that reached on polished stainless-steel. The films have a good stability in air over periods of $1 \mathrm{~h}$. On a microscopic scale of $3-30 \mu \mathrm{m}$, the films exhibit nonuniform emission properties correlated with variations in the chemical composition.
\end{abstract}

Presented at the Wire Chamber Conference, Vienna, February, 1995.

To be published in Nuclear Instruments and Methods in Physics Research A

* Corresponding author 


\section{Introduction}

CsI photocathodes have been proven to be reliable and efficient UV convertors in gaseous photon imaging detectors. Considerable experience has been gained with such devices in small- and medium-size detector prototypes [1], including RICH devices $[2,3,4]$. In view of the growing interest in large-area UV-photon imaging devices of this kind for the fast readout of scintillators and of Cherenkov light, it is important to find reliable technologies for production of large CsI surfaces with uniform and stable high quantum efficiency (QE). The scaling up in size is not trivial as it often involves a change of important parameters such as the photocathode substrate material, method of production and handling, and the conditions under which the photocathode is operated (detector construction materials, gas purity, etc.) Each of these could affect the surface quality of the photocathode and its quantum efficiency.

We report here on the results of our recent systematic studies of the $\mathrm{QE}$ and the surface structure of CsI films prepared by vacuum evaporation. The experimental techniques used include laterally resolved methods such as SEM and AFM (scanning electronic and atomic force microscopy), XSEM (X-ray secondary electron microscopy), ESCA (electron spectroscopy for chemical analysis, with secondary and primary electron detection) and PEEM (photoemission electron emission microscopy) with a deuterium UV source and Synchrotron Radiation. Integral methods were also used, as for example X-ray diffraction and $\mathrm{QE}$ measurements in current mode in vacuum, using a UV-monochromator $(140-220 \mathrm{~nm})$ and irradiating sample surfaces of $\approx 3 \mathrm{~cm}^{2}$. RICH devices, incorporating $\mathrm{UV}$ detectors with photocathodes having an area of $30 \times 30 \mathrm{~cm}^{2}$ were also used to deduce the absolute QE of CsI [5] under gas multiplication in gaseous detectors environment.

Many samples were characterized, vacuum deposited on substrates of polished stainless steel (SS), copper, aluminium, silicon, and printed circuit boards (PCB). Some of the substrates were coated with additional thin intermediate films (carbon, aluminium) under the CsI. 
The following is a short summary of the results of our study. More detailed information may be found in references $4,6,7$ and in following articles.

\section{Film topology and chemical structure}

Using SEM and AFM the structure of evaporated CsI layers (typically 200-500 $\mathrm{nm}$ thick) was studied. It was found that when smooth substrates such as $\mathrm{Si}$ or polished metal (Al, SS) are used, the layer exhibits a uniform polycrystalline structure. The average grain size varies between 0.3 to $3 \mu \mathrm{m}$, and it depends mainly on the nature of the surface on which the layer is deposited. Fig. 1 displays a typical aspect of a CsI layer, evaporated on C-coated Si. The grain size decreases with increasing $\mathrm{C}$ thickness, probably due to the increased roughness of the surface. Similar results were obtained with Al-coated Si. Each grain seems to contain $\mathrm{CsI}$ in a crystalline cubic structure. Fig. 2, shows the AFM image of CsI on C-coated Si on an atomic scale, together with a Fourier transform of the image. The regular pattern reflects the crystalline structure. From X-ray diffraction studies of CsI on $\mathrm{Al}$ [6], the lattice parameter value was extracted, in good agreement with the literature.

A completely different structure was found [5] when CsI was evaporated on a standard PCB, in which the copper readout pads were covered with a chemically deposited $\mathrm{Au}$ layer (standard industrial process). In this case the evaporated layer exhibits a very rough face, with an irregular layout of grains of various shapes and sizes (fig. 3a); cracks on the surface probably indicate differences in thermal expansion coefficient; an X-ray diffraction study of this sample, particularly after its short exposure to ambient air, showed that the layer contains $\mathrm{CuI}, \mathrm{I}_{2}$ and $\mathrm{Cs}$ components, but no $\mathrm{CsI}[6]$. We conclude that the CsI chemically reacts with the $\mathrm{Cu}$ substrate, and the intermediate Au layer is ineffective in preventing this interaction.

We found that PCBs, polished and then covered with $\mathrm{Ni}$ or with $\mathrm{Ni} / \mathrm{Au}$ (industrial chemical deposition process), however, provide inert substrates, and allow for the production of uniform polycrystalline CsI films (Fig. 3b) with the typical regular granular structure observed on $\mathrm{Al}$ or $\mathrm{SS}$. It was checked, that the gross 
structure of about $3 \mu \mathrm{m}$ period (delimited by the dark areas in the figure) and the roughness of about $250 \mathrm{~nm}$ are due to the primary surface structure of the substrate. AFM imaging on an atomic scale shows a regular pattern of atoms, being identical to that of Fig. 2.

It is interesting to note that the $\mathrm{Ni}$ and $\mathrm{Ni} / \mathrm{Au}$ coated substrates not only provide a more homogenous morphology, but the CsI layers are more stable with exposure to air. The examined samples were sometimes exposed for a few hours to air, without any notable change in topology. The granular aspect of the CsI films could explain some angular photon incidence dependence of the quantum efficiency, recently observed [8]. It could also affect the surface resistivity, which is important for stable photocathode operation under gas multiplication at high photon flux.

\section{Emission inhomogeneity and correlated chemical analysis}

High resolution laterally resolved secondary emission analysis was carried out by several methods, using incident radiation ranging from UV to X-rays. A detailed description of the methods and results is given elsewhere [6-7]. We shall summarize here the main experimental observations.

All methods of analysis indicate that on a microscopic scale of $3-30 \mu \mathrm{m}$ the emission from the CsI layer is highly inhomogeneous. Fig. 4 shows ESCA secondary electron spectra taken at different positions on a heat-enhanced (see paragraph 4) $\mathrm{Al} / \mathrm{CsI}$ photocathode. The area irradiated by the X-ray beam is of the order of $20 \mathrm{x} 20$ $\mu \mathrm{m}^{2}$. Intensity differences up to a factor of 3 are observed. An interesting observation is given in Fig. 5, showing a PEEM image with $3 \mu \mathrm{m}$ lateral resolution, taken with a Synchrotron Radiation Source. The secondary electron emission image $\left(40 \times 40 \mu \mathrm{m}^{2}\right)$ on the top, shows an inhomogeneous secondary electron emission (SEE) yield distribution, with bright and dark regions, representing high and low emission regions, respectively. On the bottom, on an enlarged scale, the secondary emission is presented together with the digitally subtracted images of the primary electron emission, corresponding to $\mathrm{I} 4 \mathrm{~d}$ and $\mathrm{Cs} 4 \mathrm{~d}$ levels respectively. The enhanced secondary electron yield is correlated to regions with an excess of I. 
The same conclusion was also obtained by a similar analysis made with the ESCA [7], and with the PEEM [7] methods.

\section{Temperature enhancement of the $\mathrm{QE}$}

The temperature enhancement of the QE of CsI films on $\mathrm{Ni}$ - and $\mathrm{Ni} / \mathrm{Au}$-coated PCBs was studied in the UV with a monochromator and with Cherenkov photons. The films exhibit the same heat enhancement features in vacuum, reported for CsI on SS [9] and reach similar final QE values. This is shown in fig. 6. The nature of the enhancement process, previously observed in other conditions [10,11], is not yet understood, but we suppose that it is related to annealing of structural defects (dislocations) produced during the evaporation. The enhancement process under high vacuum (10-7 Torr) takes several hours, as shown in fig. 6. The temperature-enhanced $\mathrm{QE}$ was confirmed with a $\mathrm{RICH}$ device, which incorporated a large CsI photocathode on a $\mathrm{PCB} / \mathrm{Ni} / \mathrm{Au}$ electrode, annealed as discussed above. The $\mathrm{QE}$ values, extracted from the average number of Cherenkov photons on a ring, are very close to the best values obtained in the laboratory on SS substrates [2]. The procedure is described in detail elsewhere [2,5].

\section{Air stability}

The QE stability under exposure to ambient air was investigated by a sequence of exposures followed by pumping and measuring the $\mathrm{QE}$, in high vacuum, with the UV-monochromator system. In addition, the response of large area photocathodes of a RICH device was studied before and after exposure to air.

Fig. 7 presents the QE drop of an air-exposed (35\% relative humidity) CsI film, evaporated on a Ni/Au coated PCB. It is obvious from this figure that short term (15-30 min.) exposures do not deteriorate the $Q E$, and if there is any effect it is rapidly recovered by the pumping process.

This was not the case for CsI on standard Cu/Au PCBs. As discussed above, the dissociation of CsI leads to a permanent drop of the QE. With such photocathodes, the photon detection efficiency of the RICH was considerably inferior (x 2) to that obtained in the laboratory with the monochromator system and with SS substrates. Recent 
RICH results with $\mathrm{Ni} / \mathrm{Au}$-coated PCBs are close to the best SS CsI laboratory results [2]. A 30 minute air exposure of the large Ni-Au-coated PCB of the RICH counter, did not affect either the operation stability of the UV-detector nor its QE.

\section{Conclusions}

A systematic study of CsI evaporated films was carried out, involving laterally resolved surface properties as well as the average quantum response. It was shown that uniform deposition of polycrystalline films can be achieved by proper choice of the substrate material. The size of crystalline grains depends on the nature of the substrate. On a microscopic scale of $3-30 \mu \mathrm{m}$, the photoemission is highly inhomogeneous, and the secondary electron yield reflects not only the granular structure but also some chemical variations. A better control of the secondary emission properties of the CsI layer requires further investigation of these properties in correlation with relevant preparation parameters. In particular, the role of heat enhancement should be further investigated and possibly correlated with the chemical composition of the layer.

The (laterally averaged) QE and air stability of such layers, measured both in laboratory conditions and in a RICH device, were shown to be compatible with the demands imposed by the application to large-surface UV-sensitive gaseous detectors.

We would like to acknowledge Mr. J.M. Dalin for the SEM micrographs. The work was done within the CERN RD-26 research project. It was partially supported by the United States-Israel Binational Scientific Foundation (BSF), the Commission of the European Communities, the Israel Ministry of Science and Arts and the Mordoh Mijan de Salonique Foundation. 


\section{References}

1. Proc. of the First Workshop on RICH detectors, Bari, Italy, June, 1993, Nucl. Instr. and Meth. A343 (1994) 1-326, and references therein.

2. F. Piuz, et al., Review of the development of large area CsI photocathodes for application to RICH. These proceedings.

3. S. Korpar et al., Test of photon detectors for the HERA-B RICH, these proceedings.

4. A. Di Mauro, et al., Development of a large area advanced fast RICH detector for particle identification at the LHC operated with heavy ions, Status Report of RD26, CERN/DRDC 94-49, December, 1994.

5. A. Braem et al., Nucl. Instrum. and Meth. A343 (1994) 163 and H. Berger et al., Recent results on the properties of CsI photocathodes, presented at the conference on Frontier Detectors for Frontier Physics, La Biodola, Elba, May 1994, Nucl. Instrum. and Meth., in press.

6. J. Almeida et al., Morphological effects in the Quantum yield of Cesium Iodide, submitted to Nucl. Instrum. and Meth.

7. T. dell'Orto et al., Laterally resolved measurements of Cesium Iodide Quantum Yield, submitted to Surface Science.

8. P. Miné, et al., Incident Angle effect on the quantum efficiency of CsI photocathodes, Presented at the conference on Frontier Detectors for Frontier Physics, La Biodola, Elba, May 1994. Nucl. Instrum and Meth., in press.

9. A. Breskin, et al., New ideas in CsI-based photon detectors: wire photomultipliers and protection of the photocathodes. Presented at the IEEE Nuclear Science Symposium, Norfolk, Virginia, October, 1994, to be published in IEEE Trans. Nucl. Sci.

10. D.F. Anderson et al., Nucl. Instrum. and Meth. A323 (1992) 626.

11. H. Brauning et al., Nucl. Instrum. and Meth. A327 (1993) 369. 


\section{Figure Captions}

Fig.1 AFM images of CsI layers (500 nm thick) evaporated on C-coated Si substrates: a) $\mathrm{Si}, \mathrm{b}) 100 \AA \mathrm{C}$ on $\mathrm{Si}$, c) $800 \AA \mathrm{C}$ on $\mathrm{Si}$. The layers exhibit a regular granular structure; grain size is decreases with increase of $\mathrm{C}$ thickness, due to the increase of the substrate roughness.

Fig. 2 An atomic resolution AFM image, of CsI on C-coated Si, raw data (left) and its Fourier transform (right). A regular pattern of atoms is evident, reflecting the crystalline structure of CsI.

Fig. 3 Microscopic images of $500 \mathrm{~nm}$ thick CsI films, evaporated on different substrates: (a) SEM image on a standard PCB. (b) AFM image on a Ni/Au-coated PCB.

Fig. 4 Normalized secondary electron spectra obtained by an ESCA system from several locations on the sample, $40 \mu \mathrm{m}$ apart. Large variations in secondary electron yield are evident.

Fig. 5 Position resolved PEEM images of the secondary electron yield distribution from an annealed CsI layer on polished $\mathrm{Al}$ substrate (top). An enlarged image (bottom) presents the $2 \mathrm{D}$ position resolved distributions of secondary electrons and I4d and Cs4d photoelectrons, showing a clear correlation between the secondary emission and the presence of an excess of $I$.

Fig. 6 The results of heat enhancement of the QE of CsI on Ni-coated PCB. The annealing process at $60^{\circ} \mathrm{C}$ under vacuum for several hours increases the $\mathrm{QE}$ to values which are close to those obtained with the best CsI layers deposited on polished SS substrates.

Fig. 7 The decay of the QE of CsI films evaporated on Ni/Au-coated PCB under exposure to air, at a relative humidity of $35 \%$. 

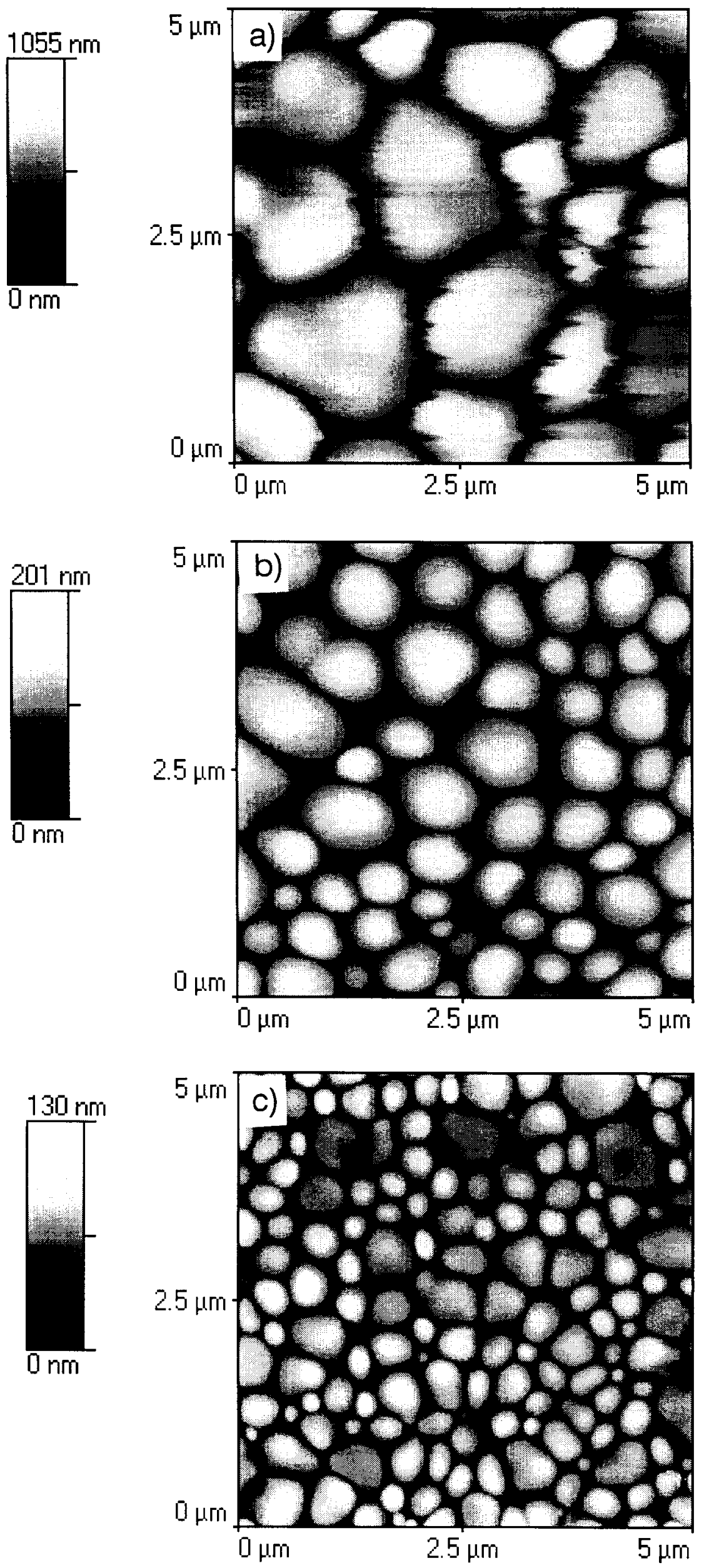

Fig. 1 

ANGSTROMS

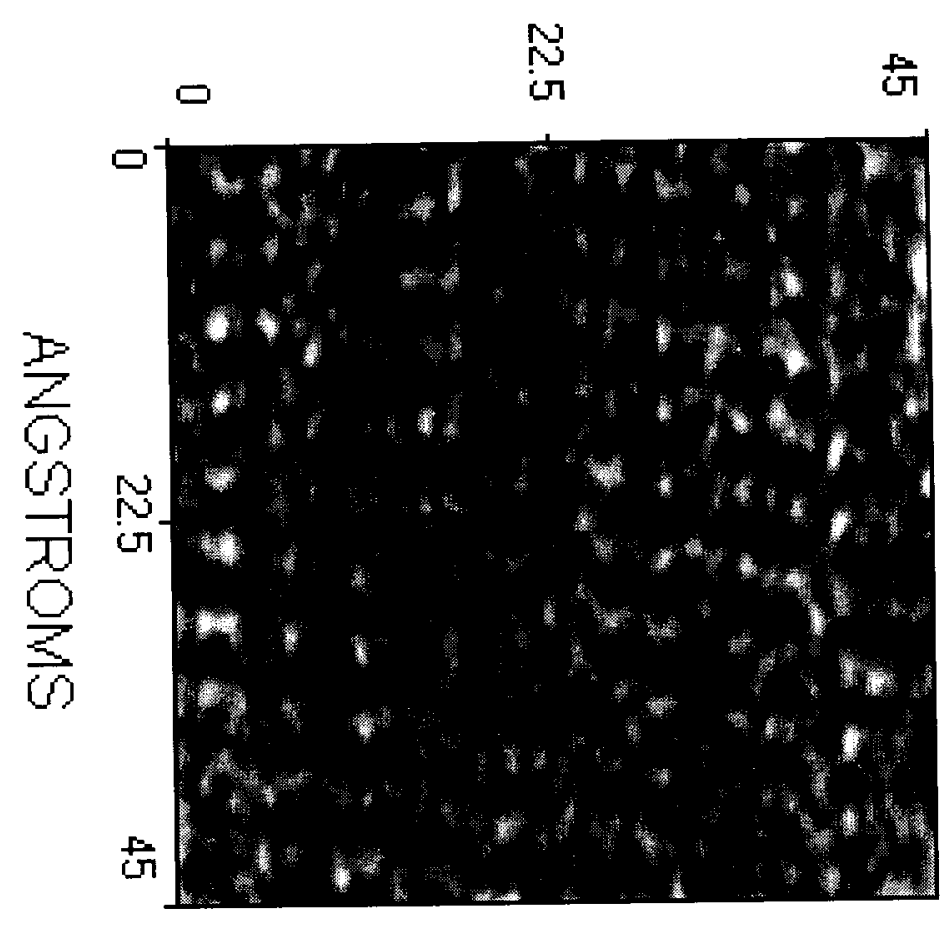

ANGSTROMS

Fig. 2

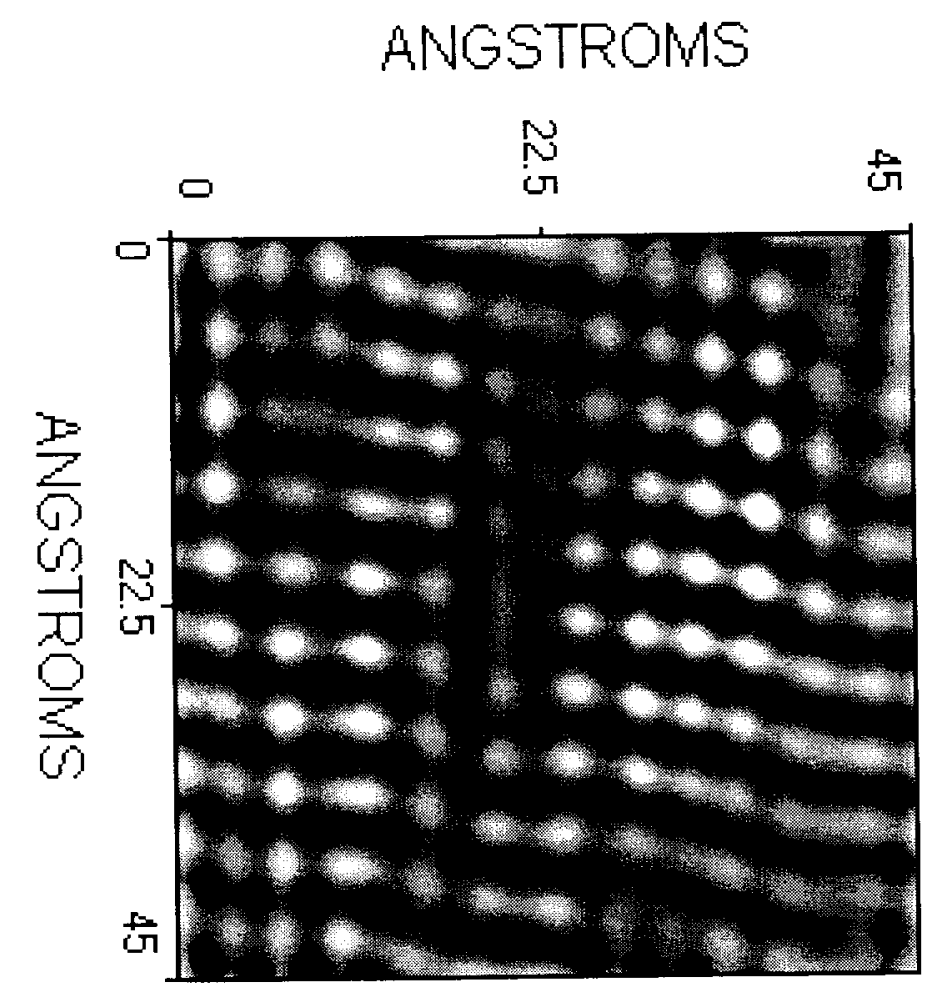




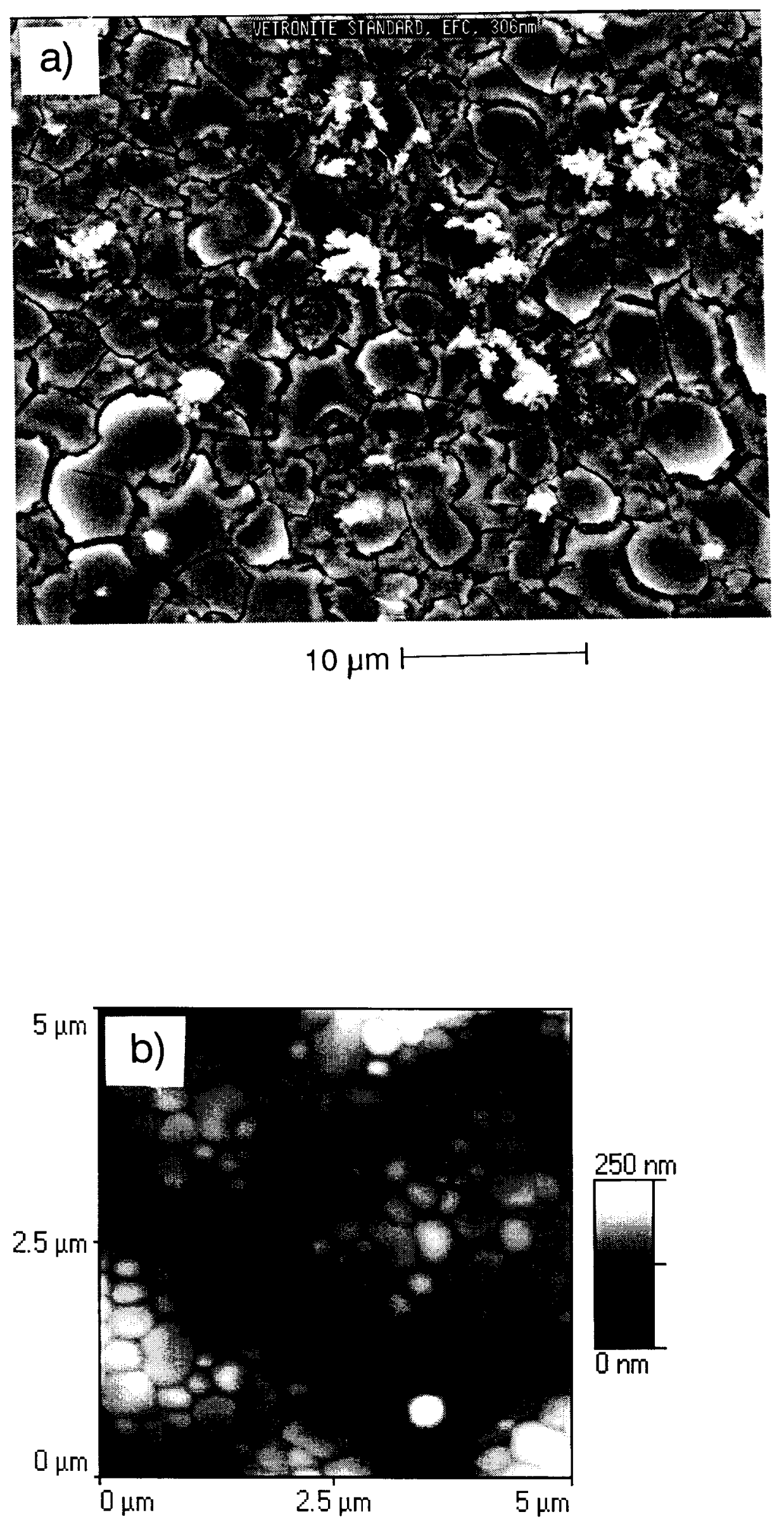

Fig. 3 



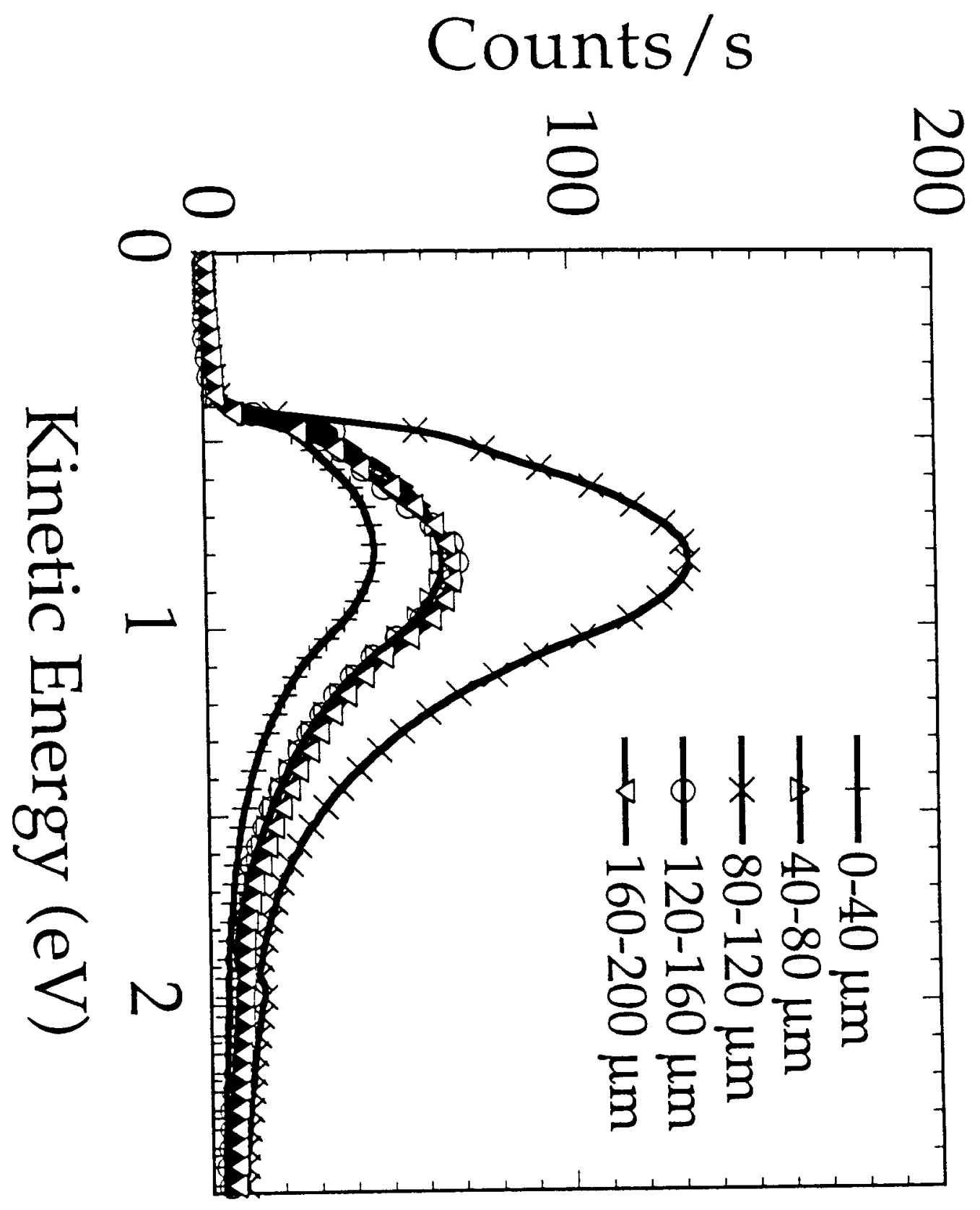

Fig. 4 



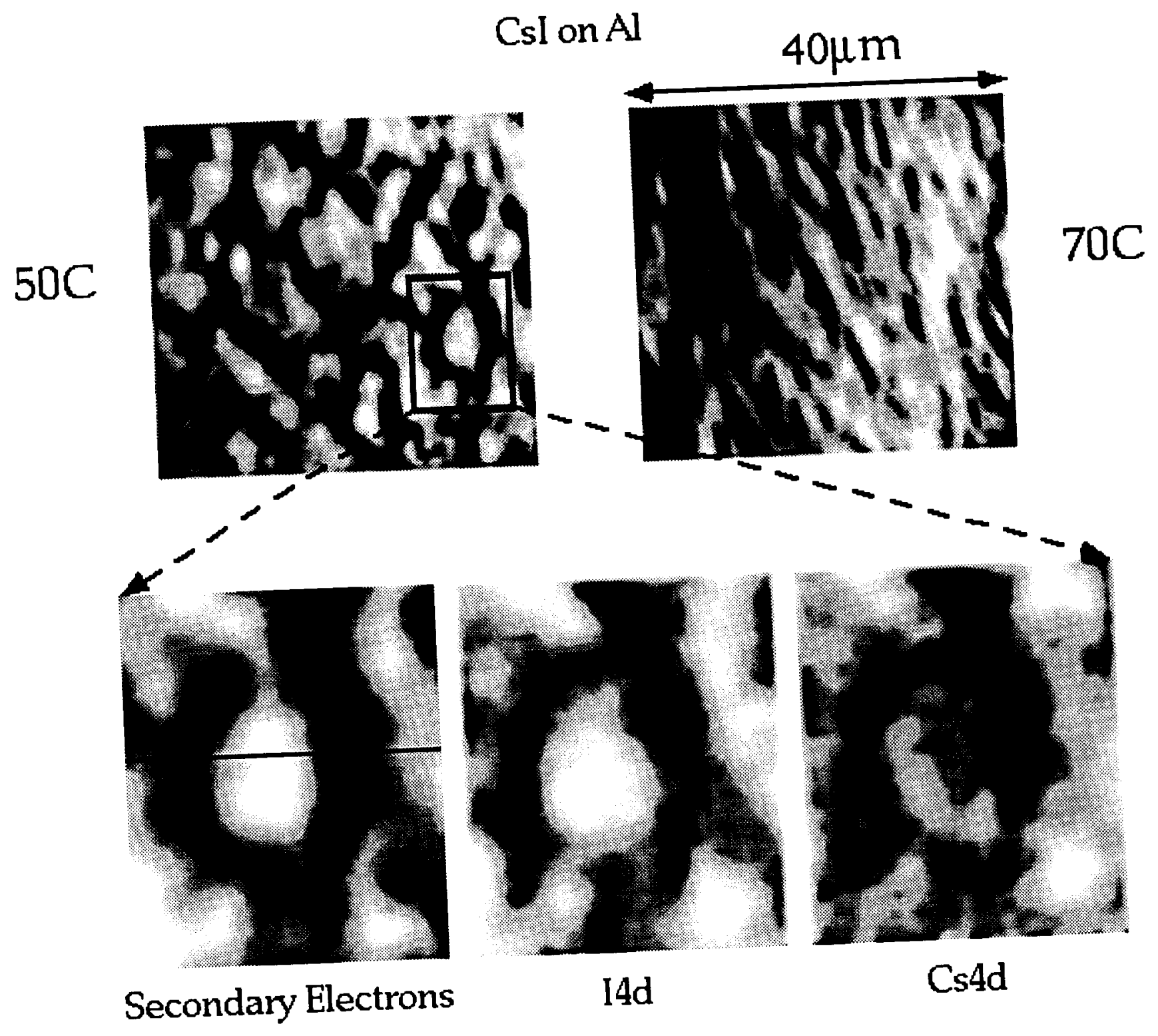

Fig. 5 



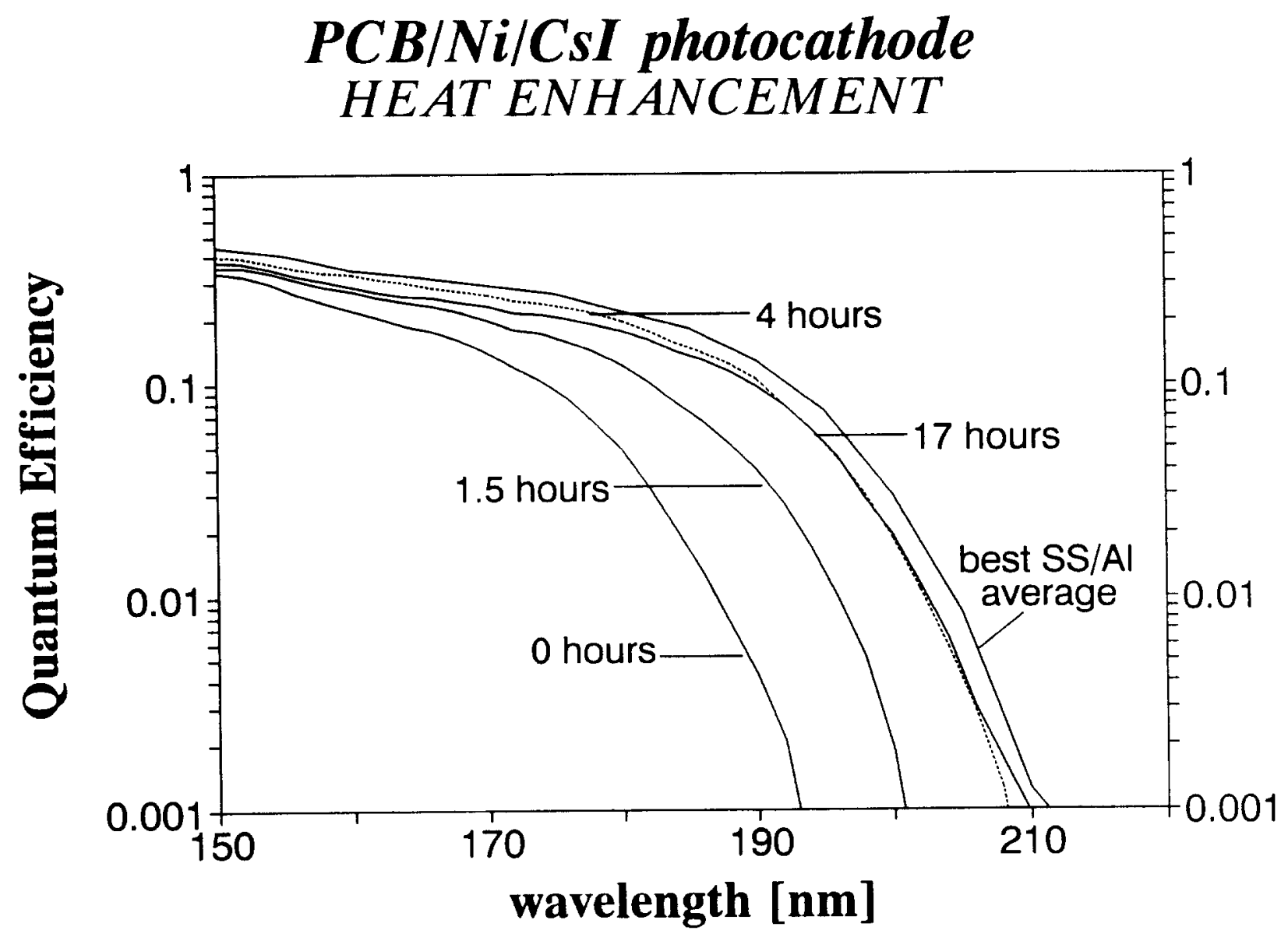

Fig. 6 



\section{PCB/Ni/Au/CsI photocathode EXPOSURE TO AIR}

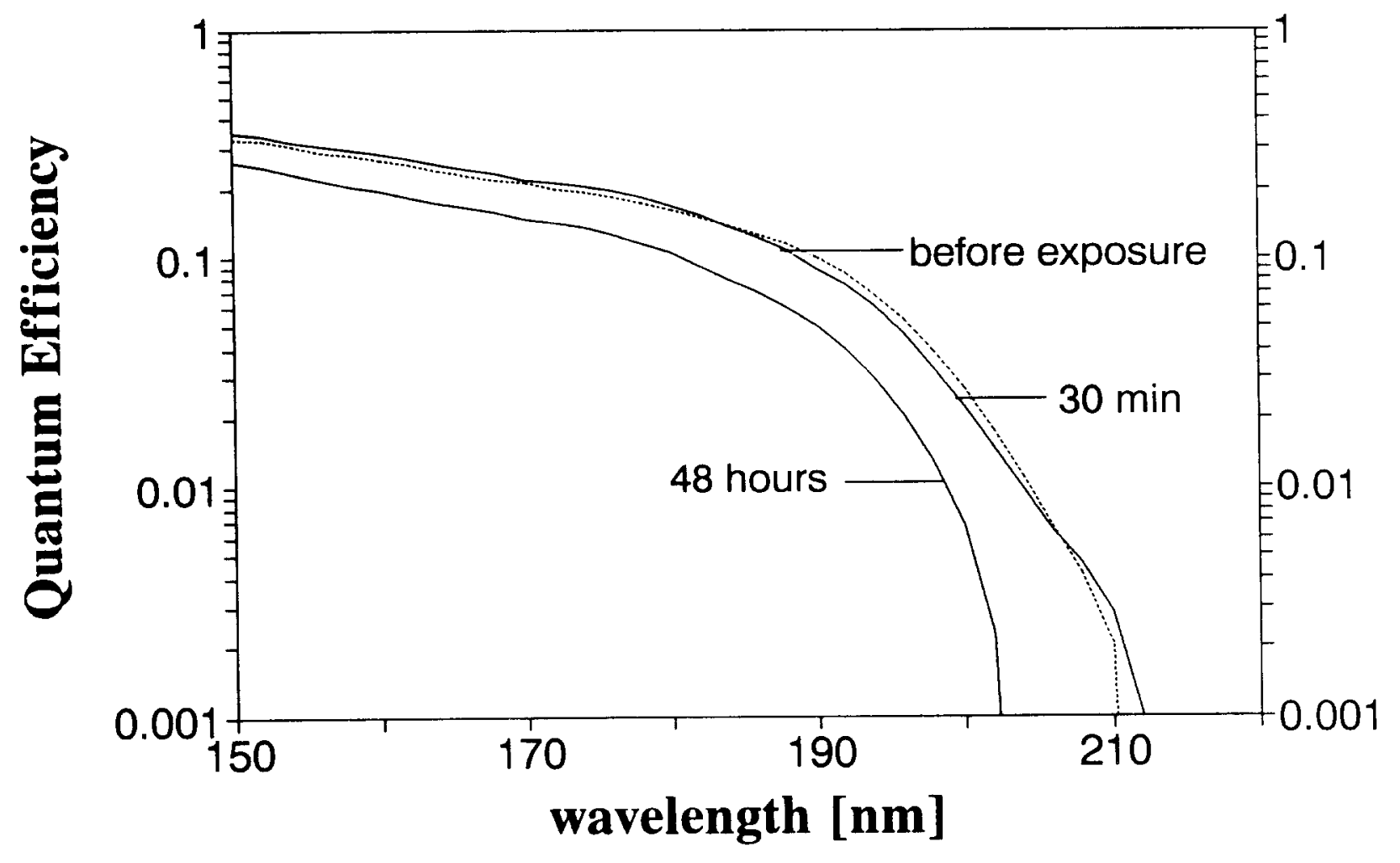

Fig. 7 
